\title{
First direct measurement of auroral and equatorial jets in the stratosphere of Jupiter
}

\author{
T. Cavalié ${ }^{1,2}$, B. Benmahi ${ }^{1}$, V. Hue ${ }^{3}$, R. Moreno ${ }^{2}$, E. Lellouch ${ }^{2}$, T. Fouchet ${ }^{2}$, P. Hartogh ${ }^{4}$, L. Rezac ${ }^{4}$, \\ T. K. Greathouse ${ }^{3}$, G. R. Gladstone ${ }^{3}$, J. A. Sinclair ${ }^{5}$, M. Dobrijevic ${ }^{1}$, F. Billebaud ${ }^{1}$, and C. Jarchow ${ }^{4}$ \\ ${ }^{1}$ Laboratoire d'Astrophysique de Bordeaux, Univ. Bordeaux, CNRS, B18N, Allée Geoffroy Saint-Hilaire, 33615 Pessac, France \\ e-mail: thibault.cavalie@u-bordeaux.fr \\ 2 LESIA, Observatoire de Paris, PSL Research University, CNRS, Sorbonne Universités, UPMC Univ. Paris 06, Univ. Paris Diderot, \\ Sorbonne Paris Cité, Meudon, France \\ 3 Southwest Research Institute, San Antonio, TX 78228, USA \\ 4 Max-Planck-Institut für Sonnensystemforschung, 37077 Göttingen, Germany \\ 5 Jet Propulsion Laboratory, California Institute of Technology, 4800 Oak Grove Drive, Pasadena, CA 91109, USA
}

Received 12 January 2021 / Accepted 6 February 2021

\section{ABSTRACT}

\begin{abstract}
Context. The tropospheric wind pattern in Jupiter consists of alternating prograde and retrograde zonal jets with typical velocities of up to $100 \mathrm{~m} \mathrm{~s}^{-1}$ around the equator. At much higher altitudes, in the ionosphere, strong auroral jets have been discovered with velocities of $1-2 \mathrm{~km} \mathrm{~s}^{-1}$. There is no such direct measurement in the stratosphere of the planet.

Aims. In this Letter, we bridge the altitude gap between these measurements by directly measuring the wind speeds in Jupiter's stratosphere.

Methods. We use the Atacama Large Millimeter/submillimeter Array's very high spectral and angular resolution imaging of the stratosphere of Jupiter to retrieve the wind speeds as a function of latitude by fitting the Doppler shifts induced by the winds on the spectral lines.

Results. We detect, for the first time, equatorial zonal jets that reside at 1 mbar, that is, above the altitudes where Jupiter's quasiquadrennial oscillation occurs. Most noticeably, we find $300-400 \mathrm{~m} \mathrm{~s}^{-1}$ nonzonal winds at 0.1 mbar over the polar regions underneath the main auroral ovals. They are in counterrotation and lie several hundred kilometers below the ionospheric auroral winds. We suspect them to be the lower tail of the ionospheric auroral winds.

Conclusions. We directly detect, for the first time, strong winds in Jupiter's stratosphere. They are zonal at low-to-mid latitudes and nonzonal at polar latitudes. The wind system found at polar latitudes may help increase the efficiency of chemical complexification by confining the photochemical products in a region of large energetic electron precipitation.
\end{abstract}

Key words. planets and satellites: individual: Jupiter - planets and satellites: atmospheres - planets and satellites: aurorae

\section{Introduction}

The tropospheric zonal wind system of Jupiter has been observed for decades showing alternating prograde and retrograde jets at the boundaries between zones and belts (Chapman 1969; Ingersoll et al. 1979, 2004; Limaye et al. 1982; García-Melendo \& Sánchez-Lavega 2001). A similar structure, although with fewer jets, has also been found in Saturn's troposphere (Smith et al. 1981; Sánchez-Lavega et al. 2000; Choi et al. 2009; Barbara \& Del Genio 2021). The mechanism behind the winds as well as their vertical extent has been extensively studied. Recent Juno and Cassini gravity field measurements have demonstrated that these winds extend down to a few thousands of kilometers below the cloud deck in Jupiter and Saturn and are therefore powered by the internal heat flux (Kaspi et al. 2018, 2020; Guillot et al. 2018; Galanti et al. 2019).

Above the tropopause, in the stratosphere, there are no tracers to infer the wind pattern from visible light imaging. The stratospheric winds could only be derived in the $20-50 \mathrm{mbar}$ and $30^{\circ} \mathrm{S}-60^{\circ} \mathrm{S}$ ranges on the exceptional occa- sion of the Shoemaker-Levy 9 (SL9) impacts from the evolution of the debris fields (Banfield et al. 1996; Sánchez-Lavega et al. 1998). The relative contributions of thermal versus mechanical forcing (by, e.g., waves and eddies) in the stratosphere are therefore unquantified. So far, the stratospheric zonal wind pattern has only been indirectly derived from the thermal wind balance relation applied to the measured zonal temperature field. There are several studies that applied this method to Jupiter and Saturn (Flasar et al. 2004, 2005; Fouchet et al. 2008; Guerlet et al. 2011, 2018; Fletcher et al. 2016; Cosentino et al. 2017). In addition, systematic infrared observations of long timescales led to the discovery of stratospheric quasi-periodic oscillations manifested in the stratospheric temperatures and winds, in particular the quasi-quadrennial oscillation (QQO) in Jupiter (Orton et al. 1991) as well as the Saturn equatorial oscillation (SEO; Orton et al. 2008). These oscillations have been the subject of numerous follow-up observations and modeling efforts to obtain robust constraints on their origin and evolution (Cosentino et al. 2017; Li \& Read 2000; Medvedev et al. 2013; Spiga et al. 2020; Bardet et al. 2021; Giles et al. 2020; 
Antuñano et al. 2020). However, deriving the wind field from the thermal wind balance is only an approximation, which in addition breaks down at the equator. The latter limitation can now be overcome thanks to a new prescription of this equation valid at equatorial latitudes (Marcus et al. 2019). Solving the thermal wind equation also requires a boundary condition, which is often taken as the cloud-top wind pattern. Furthermore, the temperature field is only interpolated between the tropopause and the middle stratosphere where it can be retrieved from hydrocarbon emissions. In any case, the thermal wind equation only gives wind shear, not the absolute wind speeds. The real magnitude of the stratospheric winds has thus remained elusive. Direct wind measurements in the stratosphere to quantify the role of thermal and mechanical forcing, and thus better constrain models of the planetary wave propagation that generates the stratospheric equatorial oscillations, are thus warranted.

With spectral resolving powers, $R=\lambda / \Delta \lambda$, exceeding $10^{6}$, heterodyne spectroscopy in the millimeter wavelength range has opened up the possibility of directly measuring frequency Doppler shifts induced by winds in spectral lines of molecular species, as originally demonstrated at Venus and Mars (Shah et al. 1991; Lellouch et al. 1991). The Atacama Large Millimeter/submillimeter Array (ALMA) now enables almost instantaneous mapping, with high sensitivity and sufficiently high spectral and angular resolutions to measure windinduced Doppler shifts in most Solar System atmospheres (e.g., Lellouch et al. 2019). At Jupiter, the main difficulty resides in measuring Doppler shifts caused by $\sim 100 \mathrm{~m} \mathrm{~s}^{-1}$ (or less) winds superimposed onto the rapid Jovian rotation $\left(12.5 \mathrm{~km} \mathrm{~s}^{-1}\right.$ at the equator). To overcome this challenge, we use the strong millimeter lines of $\mathrm{HCN}$ and $\mathrm{CO}$, two species delivered by the impacts of comet SL9 in 1994 (Lellouch et al. 1995). The SL9-derived species were expected to be homogeneously distributed in latitude at the time of our observations (e.g., Moreno et al. 2003; Cavalié et al. 2013).

\section{Observations}

We observed Jupiter with the ALMA interferometer on March 22, 2017, at 5:11-5:36 UT with 42 12-m antennas as part of the 2016.1.01235.S project. At that time, Jupiter's equatorial diameter subtended a $43.8^{\prime \prime}$ angle, the sub-Earth point latitude was $-3^{\circ}$, and the central meridian longitude (CML) ranged from $65^{\circ}$ to $80^{\circ}$ (System III). To map the whole planet at such frequencies, we had to use a mosaic of 39 pointings. Standard pointing, bandpass, amplitude, and phase calibration observations were carried out and accounted for in the data reduction we performed using CASA 4.7.2 (additional details can be found in Appendix A). The lack of short spacings with the interferometer resulted in filtering out Jupiter's extended emission (i.e., most of the disk flux) such that only the limb observations were preserved. The baselines of the interferometer ranged from 15.1 to $160.7 \mathrm{~m}$, providing an elliptical synthesized beam of $1.2^{\prime \prime}$ (eastwest) by $1^{\prime \prime}$ (north-south). This resulted in a latitudinal resolution of $\sim 3^{\circ}$ at the equator, degrading to $\sim 10^{\circ}$ close to the poles. From each spectral cube, we extracted $\sim 550$ spectra located at the planet limb (at the 1 bar level) to oversample the beam by a factor of four to five. The accumulated on-source integration time of 24 minutes enabled us to detect the $\mathrm{HCN}$ (5-4) and $\mathrm{CO}(3-2)$ emissions at $354.505 \mathrm{GHz}$ and $345.796 \mathrm{GHz}$, respectively, with signal-to-noise ratios $(\mathrm{S} / \mathrm{N})$ of $\sim 25$ per beam at the limb at spectral resolutions of $122 \mathrm{kHz}$ and $488 \mathrm{kHz}$, respectively.

\section{3. $\mathrm{HCN}$ and $\mathrm{CO}$ vertical and horizontal distributions}

The spectral lines show limited variability in terms of amplitude, but the HCN lines present some variability in terms of width. We analyzed the vertical distributions of $\mathrm{CO}$ and $\mathrm{HCN}$ as a function of latitude from the line widths. Using empirical vertical profiles of $\mathrm{CO}$ and $\mathrm{HCN}$ with a cutoff pressure $p_{0}$, below which the species have a constant mole fraction, and the radiative transfer model of Cavalié et al. (2019), we found that CO is present at $p_{0}<5$ mbar at all latitudes, whereas $\mathrm{HCN}$ is found at the same pressure levels only at the low-to-mid latitudes $\left(60^{\circ} \mathrm{S}-50^{\circ} \mathrm{N}\right)$. At higher latitudes, $\mathrm{HCN}$ is restricted to $p_{0}<0.1 \mathrm{mbar}$ (see Fig. 1). This is surprising because HCN and CO share the same origin, are both long-lived, and should thus have similar horizontal and vertical distributions. The missing spectral signature of $\mathrm{HCN}$ at pressures higher than $0.1 \mathrm{mbar}$ and at high latitudes exhibits asymmetry in latitude between the northern and the southern hemispheres: The transition between the broad $\mathrm{HCN}$ lines seen at low and mid-latitudes with the thin $\mathrm{HCN}$ lines seen in the polar region is at $60^{\circ} \mathrm{S}$ versus $50^{\circ} \mathrm{N}$. These facts point to a chemical sink for HCN related to the aurorae, the latitudinal extent of which shows similar asymmetry in latitude between the north and the south. In particular, aerosols are known to be more abundant at high latitudes (Zhang et al. 2013), suggesting adsorption of HCN on aurorally produced aerosols as a potential sink mechanism (Anderson et al. 2016).

\section{Wind speed retrieval}

Within a synthetic beam, the line is naturally Doppler-shifted by the rapid rotation of the planet. Any additional Doppler shift of the line is then indicative of atmospheric motions along the line-of-sight (LOS) located at the altitude of the wind. "Wind contribution functions", as defined by Lellouch et al. (2019), indicate that fitting the HCN line enables us to retrieve wind speeds at $\sim 1 \mathrm{mbar}$ from $60^{\circ} \mathrm{S}$ to $50^{\circ} \mathrm{N}$ and at $0.1 \mathrm{mbar}$ at polar latitudes (see Appendix B and Fig. B.1). We determined the LOS wind speeds as a function of latitude by fitting the HCN lines with a Markov chain Monte Carlo (MCMC) scheme (Goodman \& Weare 2010; Foreman-Mackey et al. 2019). We fitted all extracted limb spectra using a parametrized line shape that is fully defined by four parameters (see Appendix C for a more detailed description). The only parameter of interest is the central frequency of the line; the other three parameters are related to the width and amplitude of the line and help us to have a good rms metric for the fitting algorithm. This method is independent of any prior knowledge of the $\mathrm{HCN}$ and $\mathrm{CO}$ distributions or the atmospheric temperature. We used several hundred iterations to fit the line center position and derive its uncertainty. The altitudes of the winds are estimated from the contribution function calculations, as described above. Figure 2 (top) shows the derived LOS wind speeds we obtained from $\mathrm{HCN}$ as a function of latitude. The associated uncertainties result from the combination of the continuum subtraction on the spectra, uncertainties in the subtraction of the planet rotation associated with pointing errors, and the uncertainty of the MCMC fitting procedure (see Appendix D). The wind speeds in Fig. 2, as opposed to the zonal mean wind speeds at the cloud top found in the literature (e.g., Ingersoll et al. 2004), are measured instantaneously. The combination of the lower spectral resolution and lower $\mathrm{S} / \mathrm{N}$ of the $\mathrm{CO}$ observations does not allow us to retrieve wind speeds. We can only put a three-sigma upper limit of $150 \mathrm{~m} \mathrm{~s}^{-1}$ at $3 \mathrm{mbar}$, which is the pressure level at which winds would be measured from the fitting of the $\mathrm{CO}$ line. 

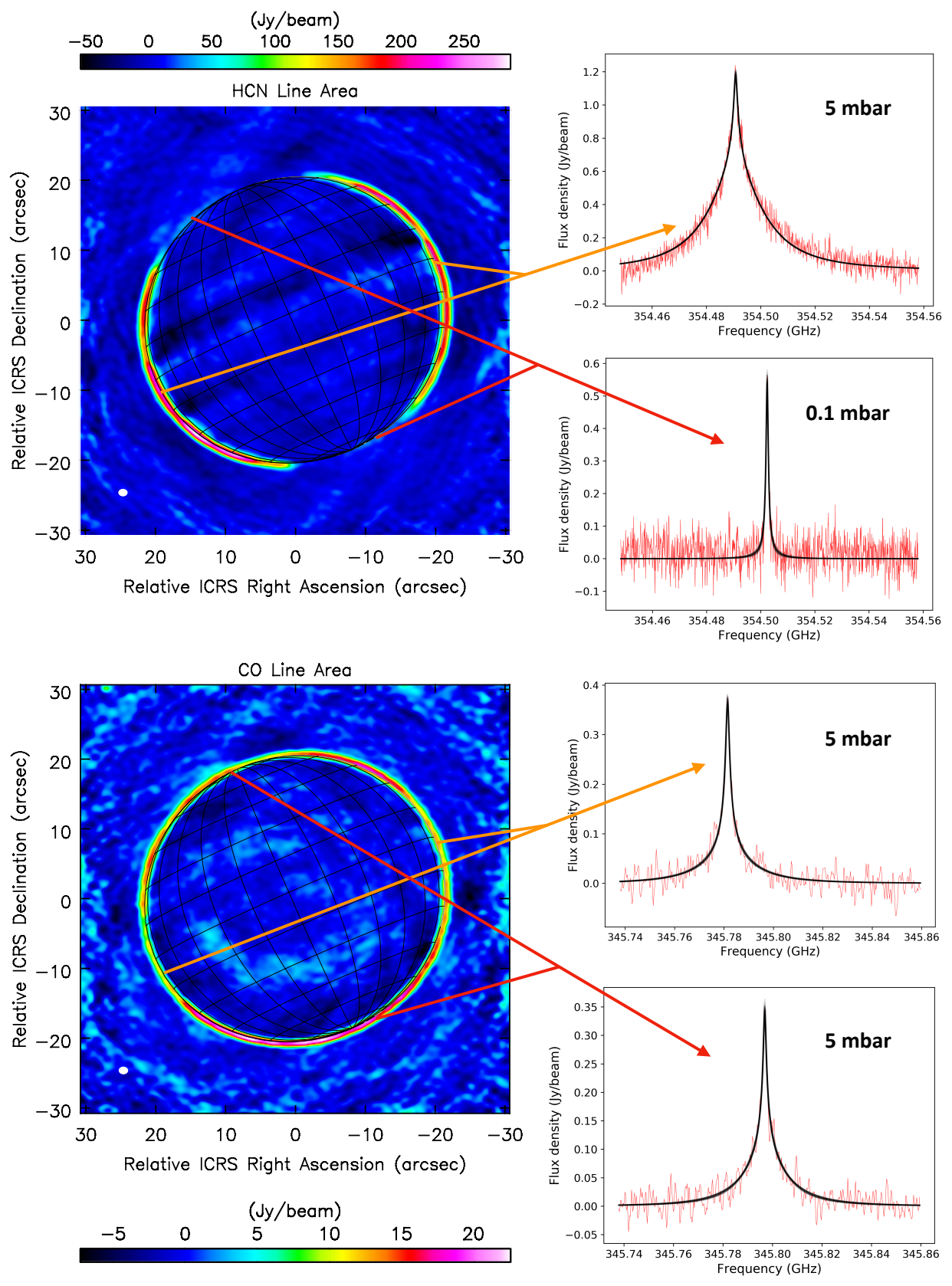

Fig. 1. ALMA observations of Jupiter's stratospheric $\mathrm{HCN}$ and CO. Left: line area maps of the $\mathrm{HCN}(5-4)$ (top) and $\mathrm{CO}$ (3-2) (bottom) emission at the limb of Jupiter. Right: spectra extracted from the data cubes (red lines), showing typical line shapes and the cutoff pressure $\left(p_{0}\right)$ in the species vertical profile to reproduce the line width, with the 30 best-fit spectra computed with the MCMC procedure from the parametrized line shape. Observable Doppler shifts with respect to the line rest frequencies are caused by the planet's rapid rotation and the local east-west winds.

\section{Results}

\subsection{Wind speed retrieval results at low-to-mid latitudes}

From $60^{\circ} \mathrm{S}$ to $50^{\circ} \mathrm{N}$, the strongest and broadest wind we detect is located at $9-11^{\circ} \mathrm{N}$, as shown in Fig. 2. It is a prograde jet with a peak LOS velocity of $+215 \pm 25 \mathrm{~m} \mathrm{~s}^{-1}$ on the planet's eastern limb and $-115 \pm 25 \mathrm{~m} \mathrm{~s}^{-1}$ on the planet's western limb. The average eastward wind speed is $165 \pm 40 \mathrm{~m} \mathrm{~s}^{-1}$ (Fig. 2, bottom), compatible with the magnitude of the near-equatorial jet found from the thermal wind balance by Flasar et al. (2004) This jet has a full width at half maximum (FWHM) of $\sim 7^{\circ}$. The difference in peak velocities between the two limbs indicates that the local vortices could accelerate or decelerate winds by $\sim 50 \mathrm{~m} \mathrm{~s}^{-1}$. This situation could thus be similar to what is seen at the cloud level, where García-Melendo et al. (2011) found that the equatorial zone shows variability in wind speeds of $\sim 20 \mathrm{~m} \mathrm{~s}^{-1}$ on aver- age (but up to $60 \mathrm{~m} \mathrm{~s}^{-1}$ ) over only one planet rotation because of vortices and planetary waves. We tentatively find a retrograde jet at $2^{\circ} \mathrm{S}$ with a $2-\sigma$ confidence level only. Its speed on the eastern limb is $-140 \pm 25 \mathrm{~m} \mathrm{~s}^{-1}$, but we cannot unambiguously identify it on the western limb. The presence of a prograde jet at $4-7^{\circ} \mathrm{S}$ is even more tentative $(1.5-\sigma)$. The equatorial wind structure at 1 mbar is thus asymmetrical with respect to the equator, contrary to the cloud-top wind structure and contrary to what one would expect in the QQO altitude and latitude ranges. It may result from the latitudinal temperature gradients found between the upper stratospheric layers and the millibar region where the QQO occurs (Cosentino et al. 2017); these gradients were also found to be asymmetrical at the time of our observations (Giles et al. 2020). In the northern and southern low-to-mid latitude, there is little evidence of other jets outside the equatorial region. 
Winds from HCN(5-4)
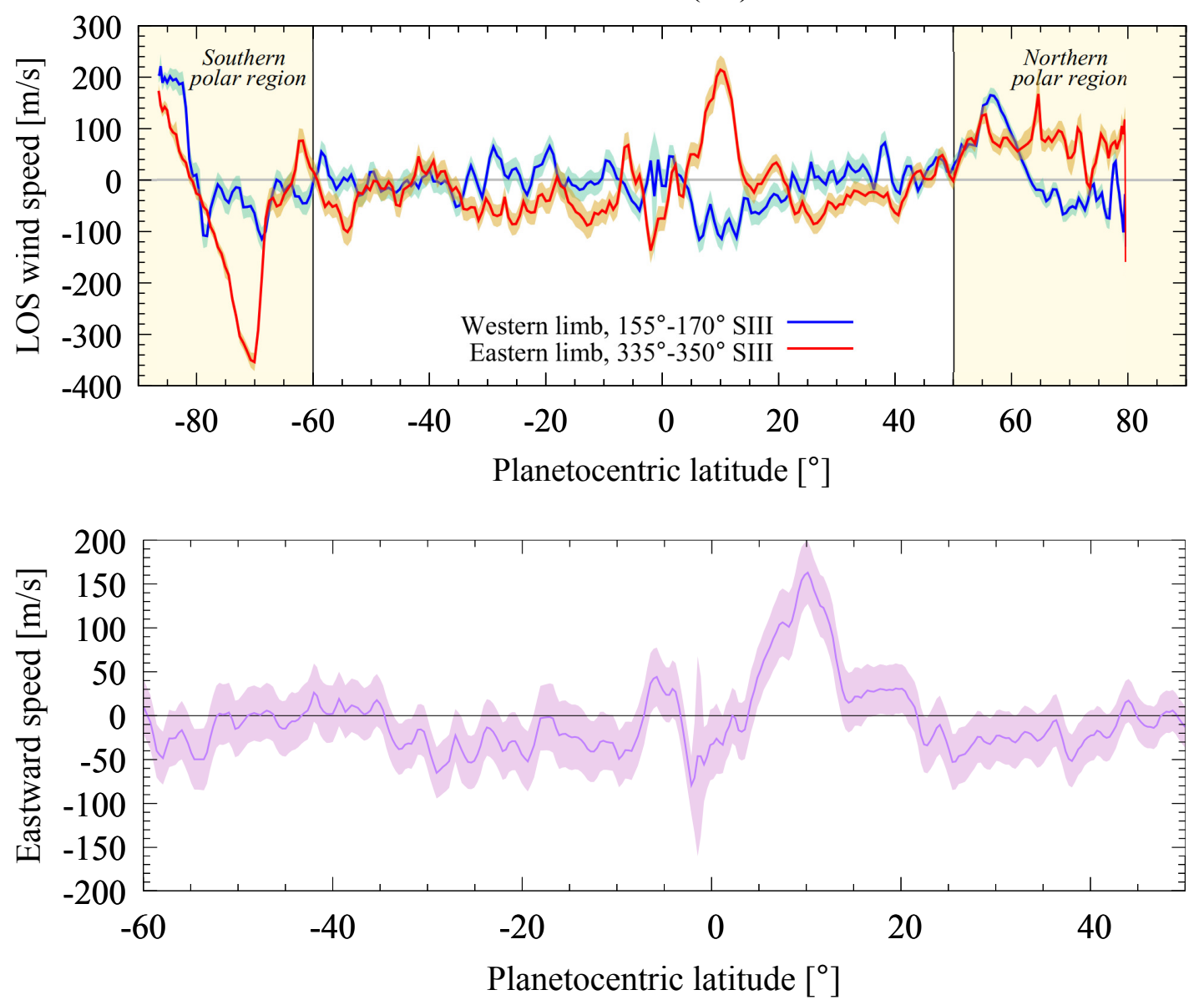

Fig. 2. Jupiter's stratospheric winds. Top: instantaneous LOS wind speed measurements as a function of latitude obtained from ALMA spectral mapping observations of the $\mathrm{HCN}(5-4)$ line. The winds are measured at $1 \mathrm{mbar}$ from $60^{\circ} \mathrm{S}$ to $50^{\circ} \mathrm{N}$ and at 0.1 mbar at polar latitudes. The western limb data are plotted in blue and the eastern limb data in red (1- $\sigma$ uncertainty envelopes in light blue and orange, respectively). Bottom: eastward wind speeds averaged from both limbs from $60^{\circ} \mathrm{S}$ to $50^{\circ} \mathrm{N}$. Prograde winds have positive speed values.

\subsection{Wind speed retrieval results in the polar regions}

The most unexpected and outstanding features detected in our observations are the nonzonal winds seen in the northern and southern polar regions (see Fig. 2, top). We detect jets at 0.1 mbar at $55^{\circ} \mathrm{N}$ and $85^{\circ} \mathrm{S}$ on the western limb as well as at $70^{\circ} \mathrm{S}$ on the eastern limb. They all have counterrotation velocities. The strongest one, seen at $70^{\circ} \mathrm{S}$ on the eastern limb, has an FWHM of $7^{\circ}$ and a peak LOS velocity of $-350 \pm 20 \mathrm{~m} \mathrm{~s}^{-1}$. The wind seen at $85^{\circ} \mathrm{S}$ on the western limb peaks at $+200 \pm 20 \mathrm{~m} \mathrm{~s}^{-1}$. These peaks seem to be collocated with the position of the southern auroral oval for the CML of our observations when compared with the position of the statistical emission of the aurorae (Clarke et al. 2009) and with the $M=30$ footprints of the Connerney et al. (2018) model of the magnetic field (i.e., the footprints of field lines that reach 30 Jupiter radii at the equator). The latter is a good marker of the position of the main ovals as observed by Juno's Ultraviolet Spectrograph (UVS; Gladstone et al. 2017). This comparison can be seen qualitatively in Fig. 3. The wind peaks at $70^{\circ} \mathrm{S}$ on the eastern limb and at $85^{\circ} \mathrm{S}$ on the western limb would then result from the same jet. To confirm this finding, we implemented a model in which we assumed a constant wind within the southern oval and no wind outside the oval. We took the inner and outer oval edges as defined by Bonfond et al. (2012). We simulated spectra at infinite spatial and spectral reso- lutions, Doppler-shifted them according to the LOS auroral oval wind component after carefully accounting for the geometry of the observations, and finally convolved them to the spectral and spatial resolutions of the ALMA observations. To improve the fit, we had to extend the inner and outer edges of the southern oval by $\sim 2^{\circ}$. This model demonstrates that a $370 \mathrm{~m} \mathrm{~s}^{-1}$ counterrotation wind inside the auroral oval results in asymmetric components, as observed at $70^{\circ} \mathrm{S}$ and $85^{\circ} \mathrm{S}$ (see Appendix E and Fig. E.1). However, this simple model is unable to properly fit the wind speeds within the entire auroral region. The real wind pattern in the auroral region is certainly more complex than in our simple model, similar to the ionospheric wind field derived by Johnson et al. (2017) from $\mathrm{H}_{3}^{+}$emission in the northern auroral region. The lack of spatial resolution prevents us from refining our model further without additional and unconstrained parametrization (e.g., variable wind speed within the oval, wind gradient at the interface between the oval and its surroundings, and winds not only limited to the oval but also inside the auroral regions).

It is noteworthy that we find hints of a similar counterrotation jet in the northern auroral region with peak LOS velocities of $+165 \pm 15 \mathrm{~m} \mathrm{~s}^{-1}$ and an FWHM of $6^{\circ}$ in latitude at $57^{\circ} \mathrm{N}$ on the western limb. The northern oval was just coming into view at the time of the observations, thus severely limiting the viewing of the northern auroral region. A significant part of the main oval 


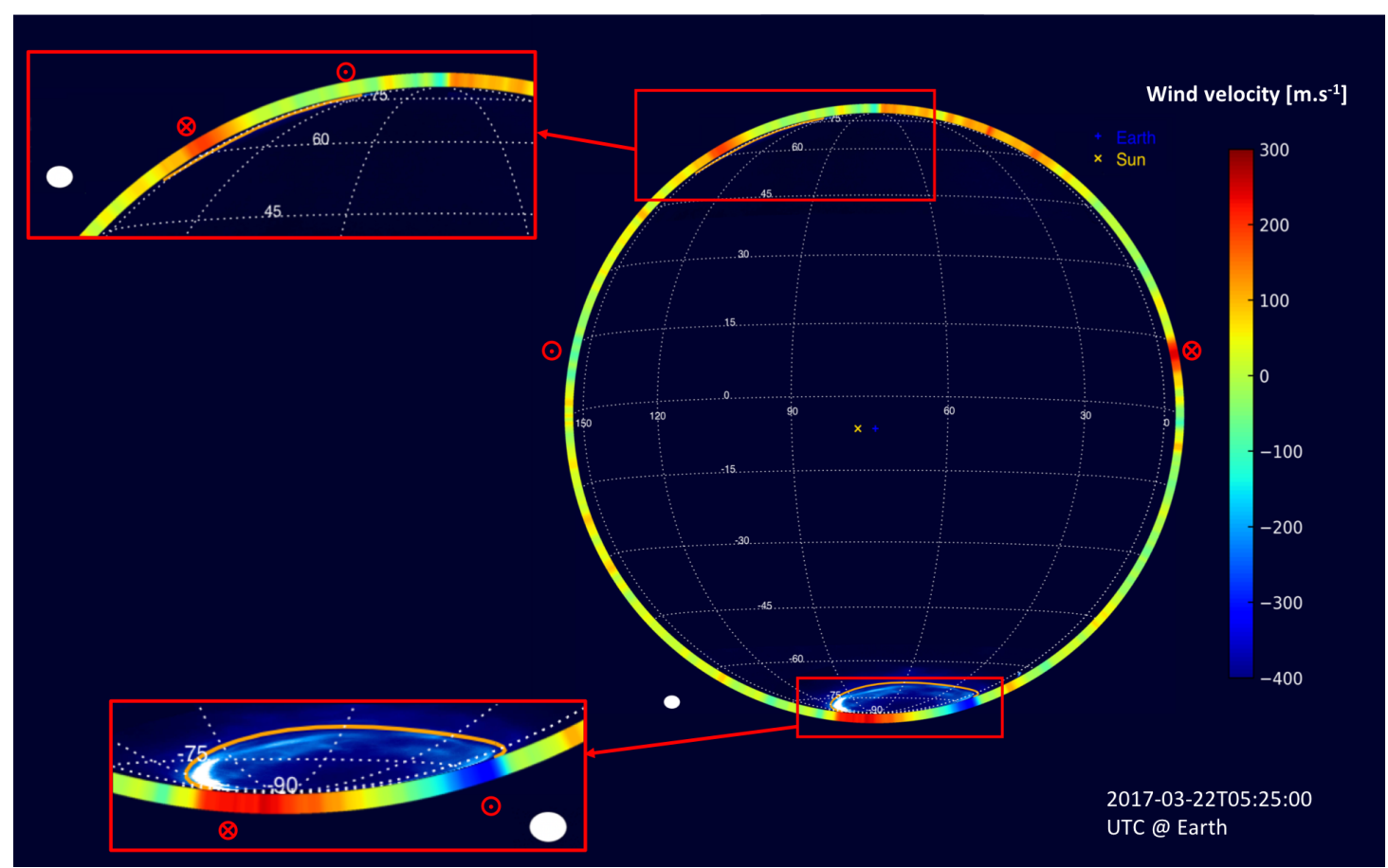

Fig. 3. Jupiter's UV aurora and stratospheric HCN winds. This composite image shows the LOS wind velocities (in $\mathrm{m} \mathrm{s}^{-1}$ ) derived from the ALMA observations and the statistical emission of the aurorae (Clarke et al. 2009) in the configuration of the ALMA observations. The northern and southern aurora regions are best seen in the dedicated zoomed-in quadrants. The $M=30$ footprints of the magnetic field model from Connerney et al. (2018) are good markers of the positions of the main ovals as seen by Juno-UVS (Gladstone et al. 2017) and are plotted in orange. The white ellipses indicate the spatial resolution of the ALMA observations. The directions of the strongest winds in the equatorial and auroral regions are indicated with the red $\odot$ and $\otimes$ symbols.

was expected to be close to tangential to the limb on its poleward edge (see Appendix F and Fig. F.1). It is thus no surprise that we find no clear evidence of the jet on the northern edge of the oval. Within the framework of our simplified model, assuming a $300 \mathrm{~m} \mathrm{~s}^{-1}$ counterrotation wind inside the northern oval nonetheless provides a good fit to the measured wind speeds poleward of $55^{\circ} \mathrm{N}$ on the western limb where the northern oval was rising (see Fig. E.1). Finally, despite the northern aurora being located on the western side, mostly behind the terminator, we see a broad signal on the eastern limb at polar latitudes with an average LOS velocity of about $+100 \mathrm{~m} \mathrm{~s}^{-1}$, for which we lack a clear explanation. A more favorable observation geometry of the northern polar region is thus required to improve our understanding of the stratospheric circulation in this region.

\section{Discussion}

The branch of the northern auroral jet we tentatively detect lies below the electrojet discovered at $p<1 \mu$ bar from infrared observations of $\mathrm{H}_{3}^{+}$emission by Rego et al. (1999) and further constrained by Stallard et al. (2001) and Johnson et al. (2017). This electrojet has a near-to-supersonic velocity of $\sim 1-2 \mathrm{~km} \mathrm{~s}^{-1}$ and is in counterrotation along the main oval (Stallard et al. 2001, 2003). Achilleos et al. (2001) showed that the $\mathrm{H}_{3}^{+}$ions could accelerate the neutrals by up to $60 \%$ of their velocity through collisions between the ionosphere and the thermosphere in the ionization peak layer $(0.07-0.3 \mu \mathrm{bar})$. The upper limit set by Chaufray et al. (2011) of $1 \mathrm{~km} \mathrm{~s}^{-1}$ on the velocity of a corresponding $\mathrm{H}_{2}$ flow confirmed a smaller neutral wind velocity, in agreement with our findings. Benefiting from ideal viewing conditions (sub-Earth latitude of $0.2^{\circ} \mathrm{N}$ ), Rego et al. (1999) also detected a similar counterrotation electrojet on the main south- ern oval. Models by Majeed et al. (2016) and Yates et al. (2020) predict that neutrals have higher velocities below the southern oval than below the northern one. Although we find relatively similar velocities underneath the two ovals, our detection in the northern oval remains tentative such that we cannot conclude on the relative magnitude between the two auroral jets. This particular point thus needs to be confirmed with new observations. Majeed et al. (2016) and Yates et al. (2020) also predict that the southern jets are expected to disappear around the $\mu$ bar level. On the contrary, our data demonstrate that the neutrals are still flowing with a substantial counterrotation velocity at the sub-millibar level below the southern oval (and probably also below the northern one), that is, $\sim 900 \mathrm{~km}$ below the corresponding ionospheric winds of Rego et al. (1999) and 100-500 km below the tentative $\mathrm{H}_{2}$ flow of Chaufray et al. (2011). Despite the strong signal-tonoise limitations of our $\mathrm{CO}$ observations at $3 \mathrm{mbar}$, we find that the southern auroral jets are at least twice slower in the millibar range than at sub-millibar levels, possibly disappearing between the sub-millibar and the millibar levels.

The detection of these auroral vortices down to the submillibar level may bear crucial implications for Jovian atmospheric chemistry. The photolysis of $\mathrm{CH}_{4}$ at the $\mu$ bar level triggers the production of more complex hydrocarbons. The addition of energetic magnetospheric electrons, which are more abundant in the auroral region than anywhere else on the planet (Gérard et al. 2014), further favors this complex ion-neutral chemistry (Wong et al. 2003). The presence of auroral vortices down to the sub-millibar level could confine the photochemical products within this region by preventing the mixing of the material inside the oval with the material outside, thus increasing the production of heavy hydrocarbons and aerosols. Auroral chemistry probably increases the production of $\mathrm{C}_{2}$ species, as 
already observed by Sinclair et al. (2018, 2019), as well as the production of aerosols (Zhang et al. 2013). The counterrotation direction of the wind in both ovals translates into a clockwise circulation on the northern oval and counterclockwise circulation on the southern one. Such a circulation pattern, which appears to be similar to anticyclones in this respect, could induce subsidence inward of the inner edge of the auroral ovals (Yates et al. 2020). The photochemically produced species would then be transported downward and could escape the auroral region at the millibar level where the vortices could be breaking up. This increased production of aerosols coupled to the downward motion could also result in the removal of HCN by adsorption onto the aerosol particles at pressures higher than $0.1 \mathrm{mbar}$ at auroral latitudes, as shown by our data. This adsorption mechanism was proposed for Titan by Anderson et al. (2016) and needs to be quantified under Jovian auroral conditions. Another effect of the downward motions would be adiabatic heating around the vortex break-up level. Heating at the millibar level was observed inside both ovals by Sinclair et al. (2017) and could be an indication that this is actually the level at which the vortices break up. We note that the independence of this heating with respect to solar illumination conditions (Sinclair et al. 2017) seems to disqualify aerosol heating as a cause. We see a sharp HCN emission increase in our data at the edges of the oval, and it could indeed be proof of such heating between the oval and its surrounding region. However, the HCN line is not optically thick, and we cannot waive the degeneracy between a temperature and an abundance increase.

The detection of stratospheric auroral jets in this work demonstrates that the Jovian atmospheric circulation is complex not only in the equatorial region owing to the QQO (Cosentino et al. 2017; Giles et al. 2020; Antuñano et al. 2020), but also in its polar regions. Repeated observations with the northern aurora in the field-of-view are necessary for a better characterization of the counterrotation stratospheric jet underneath the main oval, similar to the situation witnessed in the south.

Acknowledgements. The authors thank P. Gratier for helping implement the MCMC method, and R. Johnson and T. Stallard for providing them with their infrared ionospheric auroral wind velocities. T. C. acknowledges funding from CNES and the Programme National de Planétologie (PNP) of CNRS/INSU. This Letter makes use of the following ALMA data: ADS/JAO.ALMA\#2016.1.01235.S. ALMA is a partnership of ESO (representing its member states), NSF (USA) and NINS (Japan), together with NRC (Canada), MOST and ASIAA (Taiwan), and KASI (Republic of Korea), in cooperation with the Republic of Chile. The Joint ALMA Observatory is operated by ESO, AUI/NRAO and NAOJ.

\section{References}

Achilleos, N., Miller, S., Prangé, R., Millward, G., \& Dougherty, M. K. 2001, New J. Phys., 3, 3

Anderson, C. M., Samuelson, R. E., Yung, Y. L., \& McLain, J. L. 2016, Geophys. Res. Lett., 43, 3088

Antuñano, A., Cosentino, R. G., Fletcher, L. N., et al. 2020, Nat. Astron., 5, 71

Banfield, D., Gierasch, P. J., Squyres, S. W., et al. 1996, Icarus, 121, 389

Barbara, J. M., \& Del Genio, A. D. 2021, Icarus, 354, 114095

Bardet, D., Spiga, A., Guerlet, S., et al. 2021, Icarus, 354, 114042

Bonfond, B., Grodent, D., Gérard, J. C., et al. 2012, Geophys. Res. Lett., 39, L01105

Cavalié, T., Feuchtgruber, H., Lellouch, E., et al. 2013, A\&A, 553, A21

Cavalié, T., Hue, V., Hartogh, P., et al. 2019, A\&A, 630, A87

Chapman, C. R. 1969, J. Atmos. Sci., 26, 986
Chaufray, J. Y., Greathouse, T. K., Gladstone, G. R., et al. 2011, Icarus, 211, 1233

Choi, D. S., Showman, A. P., \& Brown, R. H. 2009, J. Geophys. Res., 114, E04007

Clarke, J. T., Nichols, J., Gérard, J. C., et al. 2009, J. Geophys. Res., 114, A05210 Connerney, J. E. P., Kotsiaros, S., Oliversen, R. J., et al. 2018, Geophys. Res. Lett., 45, 2590

Cosentino, R. G., Morales-Juberías, R., Greathouse, T., et al. 2017, J. Geophys. Res., 122, 2719

de Pater, I., Sault, R. J., Moeckel, C., et al. 2019, AJ, 158, 139

Flasar, F. M., Kunde, V. G., Achterberg, R. K., et al. 2004, Nature, 427, 132

Flasar, F. M., Achterberg, R. K., Conrath, B. J., et al. 2005, Science, 307, 1247

Fletcher, L. N., Irwin, P. G. J., Achterberg, R. K., Orton, G. S., \& Flasar, F. M. 2016, Icarus, 264, 137

Foreman-Mackey, D., Farr, W., Sinha, M., et al. 2019, J. Open Sour. Softw., 4, 1864

Fouchet, T., Guerlet, S., Strobel, D. F., et al. 2008, Nature, 453, 200

Galanti, E., Kaspi, Y., Miguel, Y., et al. 2019, Geophys. Res. Lett., 46, 616

García-Melendo, E., \& Sánchez-Lavega, A. 2001, Icarus, 152, 316

García-Melendo, E., Arregi, J., Rojas, J. F., et al. 2011, Icarus, 211, 1242

Gérard, J. C., Bonfond, B., Grodent, D., et al. 2014, J. Geophys. Res., 119, 9072

Giles, R. S., Greathouse, T. K., Cosentino, R. G., Orton, G. S., \& Lacy, J. H. 2020, Icarus, 350, 113905

Gladstone, G. R., Persyn, S. C., Eterno, J. S., et al. 2017, Space Sci. Rev., 213, 447

Goodman, J., \& Weare, J. 2010, Commun. Appl. Math. Comput. Sci., 5, 65

Grodent, D., Clarke, J. T., Kim, J., Waite, J. H., \& Cowley, S. W. H. 2003, J. Geophys. Res., 108, 1389

Guerlet, S., Fouchet, T., Bézard, B., Flasar, F. M., \& Simon-Miller, A. A. 2011, Geophys. Res. Lett., 38, L09201

Guerlet, S., Fouchet, T., Spiga, A., et al. 2018, J. Geophys. Res., 123, 246

Guillot, T., Miguel, Y., Militzer, B., et al. 2018, Nature, 555, 227

Ingersoll, A. P., Beebe, R. F., Collins, S. A., et al. 1979, Nature, 280, 773

Ingersoll, A. P., Dowling, T. E., Gierasch, P. J., et al. 2004, in Dynamics of Jupiter's Atmosphere, eds. F. Bagenal, T. E. Dowling, \& W. B. McKinnon, 1,105

Johnson, R. E., Stallard, T. S., Melin, H., Nichols, J. D., \& Cowley, S. W. H. 2017, J. Geophys. Res., 122, 7599

Kaspi, Y., Galanti, E., Hubbard, W. B., et al. 2018, Nature, 555, 223

Kaspi, Y., Galanti, E., Showman, A. P., et al. 2020, Space Sci. Rev., 216, 84

Lellouch, E., Goldstein, J. J., Bougher, S. W., Paubert, G., \& Rosenqvist, J. 1991, ApJ, 383, 401

Lellouch, E., Paubert, G., Moreno, R., et al. 1995, Nature, 373, 592

Lellouch, E., Gurwell, M. A., Moreno, R., et al. 2019, Nat. Astron., 3, 614

Li, X., \& Read, P. L. 2000, Planet. Space Sci., 48, 637

Limaye, S. S., Revercomb, H. E., Sromovsky, L. A., et al. 1982, J. Atmos. Sci., 39,1413

Majeed, T., Bougher, S. W., Ridley, A. J., et al. 2016, J. Geophys. Res., 121, 4647 Marcus, P. S., Tollefson, J., Wong, M. H., \& Pater, I. D. 2019, Icarus, 324, 198

Medvedev, A. S., Sethunadh, J., \& Hartogh, P. 2013, Icarus, 225, 228

Moreno, R., Marten, A., Matthews, H. E., \& Biraud, Y. 2003, Planet. Space Sci., 51,591

Orton, G. S., Friedson, A. J., Caldwell, J., et al. 1991, Science, 252, 537

Orton, G. S., Yanamandra-Fisher, P. A., Fisher, B. M., et al. 2008, Nature, 453, 196

Rego, D., Achilleos, N., Stallard, T., et al. 1999, Nature, 399, 121

Sánchez-Lavega, A., Gómez, J. M., Rojas, J. F., et al. 1998, Icarus, 131, 341

Sánchez-Lavega, A., Rojas, J. F., \& Sada, P. V. 2000, Icarus, 147, 405

Shah, K. P., Muhleman, D. O., \& Berge, G. L. 1991, Icarus, 93, 96

Sinclair, J. A., Orton, G. S., Greathouse, T. K., et al. 2017, Geophys. Res. Lett., 44,5345

Sinclair, J. A., Orton, G. S., Greathouse, T. K., et al. 2018, Icarus, 300, 305

Sinclair, J. A., Moses, J. I., Hue, V., et al. 2019, Icarus, 328, 176

Smith, B. A., Soderblom, L., Beebe, R. F., et al. 1981, Science, 212, 163

Spiga, A., Guerlet, S., Millour, E., et al. 2020, Icarus, 335, 113377

Stallard, T., Miller, S., Millward, G., \& Joseph, R. D. 2001, Icarus, 154, 475

Stallard, T. S., Miller, S., Cowley, S. W. H., \& Bunce, E. J. 2003, Geophys. Res. Lett., 30, 1221

Wong, A.-S., Yung, Y. L., \& Friedson, A. J. 2003, Geophys. Res. Lett., 30, 1447

Yates, J. N., Ray, L. C., Achilleos, N., Witasse, O., \& Altobelli, N. 2020

J. Geophys. Res., 125, e26792

Zhang, X., West, R. A., Banfield, D., \& Yung, Y. L. 2013, Icarus, 226, 159 


\section{Appendix A: Observations, data reduction, and imaging}

Observations of Jupiter for ALMA project 2016.1.01235.S were executed on March 22, 2017. We used 42 antennas of the 12-m telescope array. The shortest and longest baselines were 15.1 and $160.7 \mathrm{~m}$, respectively. The observations started with calibration observations between 4:46 UT and 5:11 UT. After initial pointing calibration, extended bandpass calibration observations were carried out on J1256-0547 to comply with the high spectral dynamic range required by the high $\mathrm{S} / \mathrm{N}$ observations of $\mathrm{CO}$ and $\mathrm{HCN}$ emission lines on the bright Jovian continuum. Finally, amplitude calibration observations were acquired using Ganymede as a target. Between 5:11 UT and 5:35 UT, most of the observation time was spent on Jupiter, with regular phase calibration observations on J1312-0424. Jupiter had an average elevation of $72^{\circ}$ above the horizon, and the sky transparency was adequate, with 0.85 to $0.95 \mathrm{~mm}$ of precipitable water vapor. These conditions resulted in system temperatures at 345 and $354 \mathrm{GHz}$ ranging from 120 to $190 \mathrm{~K}$ and from 140 to $240 \mathrm{~K}$, respectively. We covered the full Jovian disk with a 39-point mosaic over a square region of $60^{\prime \prime} \times 60^{\prime \prime}$ with Nyquist sampling.

The spectral setup enabled us to simultaneously record the Jovian emission of the HCN (5-4) line at $354.505 \mathrm{GHz}$ and the $\mathrm{CO}(3-2)$ line at $345.796 \mathrm{GHz}$. The $\mathrm{HCN}$ line was observed in two basebands with different bandwidths and spectral resolutions. The highest spectral resolution was $122.070 \mathrm{kHz}$ $\left(\sim 103 \mathrm{~m} \mathrm{~s}^{-1}\right)$ over a bandwidth of $234.375 \mathrm{MHz}$. The CO line was observed in a single baseband with a spectral resolution of $488.242 \mathrm{kHz}\left(\sim 426 \mathrm{~m} \mathrm{~s}^{-1}\right)$ over a bandwidth of $937.500 \mathrm{MHz}$.

The data reduction process was run under CASA 4.7.2 and included pointing, bandpass, amplitude, and phase calibrations. We also corrected for the relative radial velocity of Jupiter. We then produced continuum images in the different basebands. To produce the spectral data cubes in these basebands, we applied continuum subtraction prior to the imaging stage. Because of the limited uv-plane coverage in the short baselines, the extended emission of Jupiter is mostly filtered out and only the limb of the planet is imaged. We obtain a sensitivity of $48 \mathrm{mJy} \mathrm{beam}^{-1}$ per $122 \mathrm{kHz}$ resolution element in the HCN baseband. With a peak line intensity of $1.2 \mathrm{mJy}$ beam $^{-1}$ in the mid-to-low latitudes and peak line intensities up to $2.1 \mathrm{Jy}$ in the polar regions, we obtain detections with $\mathrm{S} / \mathrm{N}$ ranging from 25 to 50 , depending on the latitude, at $122 \mathrm{kHz}$ resolution. In the $\mathrm{CO}$ baseband, we reach a sensitivity of $17 \mathrm{mJy}$ beam $^{-1}$ per $488 \mathrm{kHz}$ resolution element and $\mathrm{S} / \mathrm{N}$ ranging from 17 to 25 .

\section{Appendix B: Contribution functions}

We used step vertical profiles in which $\mathrm{CO}$ and $\mathrm{HCN}$ have a constant mole fraction, $y_{0}$, above a cutoff pressure, $p_{0}$. To reproduce the CO lines, which are essentially very similar in amplitude and width on the limb regardless of the latitude, we set $p_{0}=5 \mathrm{mbar}$ and $y_{0}=4 \times 10^{-8}$. For $\mathrm{HCN}$, we found $p_{0}=5 \mathrm{mbar}$ from $60^{\circ} \mathrm{S}$ to $50^{\circ} \mathrm{N}$ and $p_{0}=0.1 \mathrm{mbar}$ at polar latitudes. With $y_{0}=10^{-9}$, we could fit most of the HCN lines, except within the auroral ovals where a higher stratospheric temperature or a higher value of $y_{0}$ is required. From these radiative transfer simulations performed at the spatial and spectral resolutions of the observations, we derived the contribution functions of the $\mathrm{CO}$ and $\mathrm{HCN}$ lines at the limb, both at the line centers and in their wings (at $10 \mathrm{MHz}$ from the line center). The results shown in Fig. B.1A indicate that the $\mathrm{CO}$ line center is formed at $0.5-5$ mbar levels, while its wings are formed around the 5 mbar level. We found that the
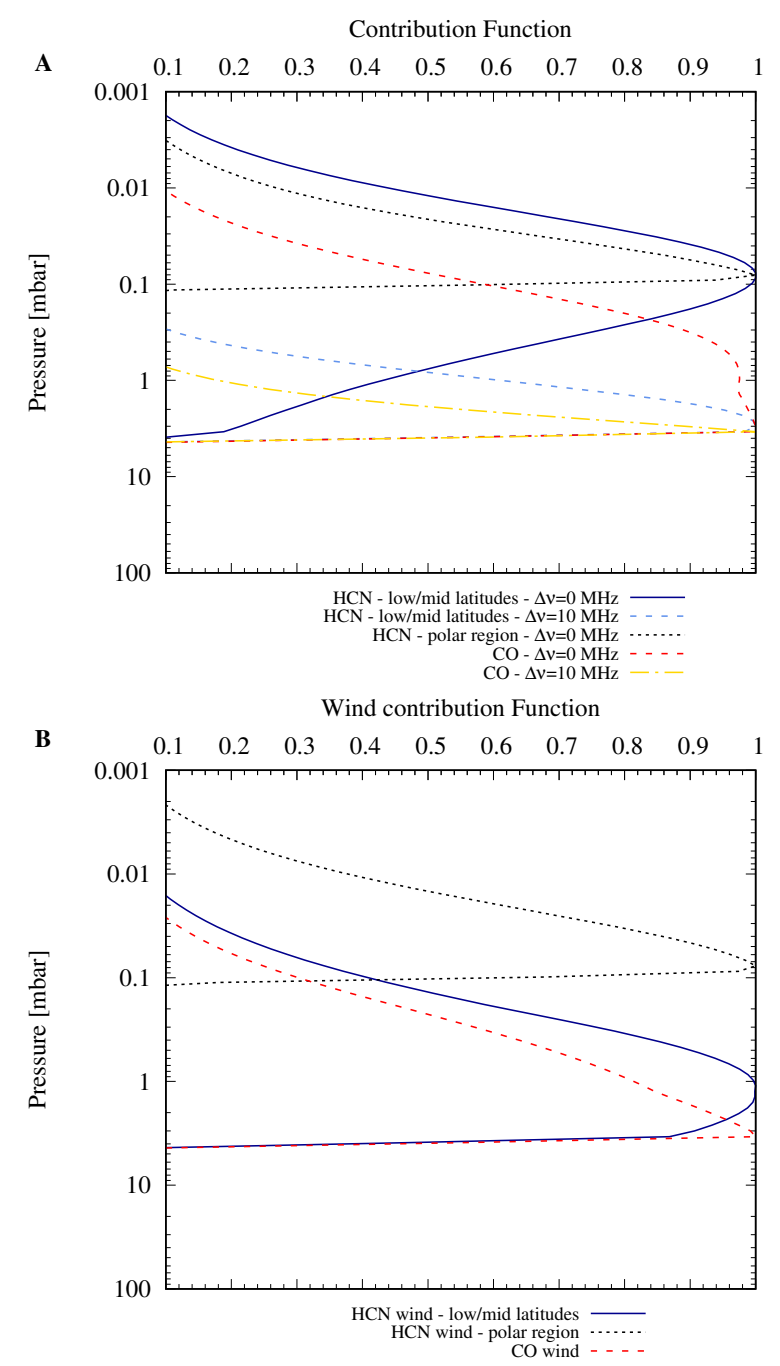

Fig. B.1. Contribution and wind contribution functions. $A$ : contribution functions of the HCN and CO lines at Jupiter's limb at the spectral and spatial resolutions of the observations. They are computed for different frequency offsets from the line center. For $\mathrm{HCN}$, different locations (low-to-mid latitudes and polar regions) are presented. For CO, the contribution functions are similar regardless of the latitude. $B$ : wind contribution functions for $\mathrm{HCN}$ at low-to-mid (solid blue line) and polar latitudes (dotted black line) and for CO at any latitude (dashed red line).

HCN line center is formed at $\sim 0.1$ mbar regardless of the latitude. Outside polar latitudes, the line is much broader and its wings probe down to the 5 mbar cutoff level, similar to CO.

Following Lellouch et al. (2019), we computed the wind contribution function of the HCN line to establish the levels probed by the winds (see Fig. B.1B). We found that the HCN line enables measuring winds at $\sim 1$ mbar from $60^{\circ} \mathrm{S}$ to $50^{\circ} \mathrm{N}$, and at $\sim 0.1$ mbar at polar latitudes. The CO line would, in principle, allow us to measure winds at 3 mbar; however, the $\mathrm{S} / \mathrm{N}$ of the observations is insufficient given the spectral resolution, and we can only set an upper limit on the wind speed at this pressure level.

\section{Appendix C: Retrieval of wind speeds as a function of latitude}

Given the spatial resolution of our observations, spectral lines should be asymmetric in the case of a vertically varying wind 
profile. However, we note that the $\mathrm{S} / \mathrm{N}$ is not sufficient to derive a full vertical wind profile. We therefore assume a vertically constant wind in the altitude layers where the lines are formed and study the meridional variability of the winds. We used a symmetrical parametrized line shape to fit the observations with an MCMC procedure. The profile is the following:

$$
\begin{aligned}
f\left(A, v, v_{0}, \alpha, \beta, \gamma, \delta, \Gamma\right)=A\left(\frac{1}{\sigma \sqrt{2 \pi}} \mathrm{e}^{-\frac{1}{2}\left(\frac{v-v_{0}}{\sigma}\right)^{2}}\right)^{\delta} \\
\times\left(\frac{2}{\pi \Gamma} \frac{1}{1+\left(\frac{v-v_{0}}{\Gamma / 2}\right)^{2}}\right)^{\gamma}\left(\left|v-v_{0}\right|^{\alpha}+\frac{1}{(\beta \Gamma)^{2}+\left(v-v_{0}\right)^{2}}\right) \\
\times \frac{1}{\Gamma^{2}+\left(v-v_{0}\right)^{2}+1} .
\end{aligned}
$$

This expression is composed of four functions, and each one plays a role in shaping the line. The first is a power Gaussian that enables the reproduction of the line core in the case of a strongly convolved line. The second is a power Lorentzian meant for narrow line peaks. The third is the sum of a power absolute function and a modified Lorentzian that controls the amplitude of the line. The fourth is another modified Lorentzian to shape the line wings. The $A$ is a constant.

The line profile is controlled by a set of eight parameters, and $v$ is the frequency. Of these eight parameters, $\alpha, \beta$, and $\delta$ were fixed to $6.0,16.5$, and 2.0, respectively, and $\delta$ to $0.202 \mathrm{GHz}$, after numerical testing to obtain spectral lines with narrow peaks and narrow-to-broad wings, such as the $\mathrm{HCN}$ and $\mathrm{CO}$ lines of our dataset: $\alpha$ enables the uplifting of the line wings, $\delta$ enables the production of narrow peaks and narrow wings, and $\beta$ mostly reduces the amplitude of the peak.

The $A, v_{0}, \Gamma$, and $\gamma$ are MCMC fitting parameters as well as the spectrum noise level. Some of them have preestimated ranges: $\Gamma \in[0.0001 ; 0.1] \mathrm{GHz}, \gamma \in[0.09 ; 0.11]$, and $A$ is such that the amplitude of the line profile $f$ lies within $10 \%$ of the amplitude of the observed line. Finally, $v_{0}$ is the central frequency of the line that we want to establish, and it combines the natural central frequency of the line, the rotation of the planet, and the wind component projected on the LOS. We estimated the fitting parameters (e.g., $A$ for the line amplitude) from each line before running the MCMC procedure.

We first performed a qualitative assessment of the fitting procedure and required computational time for each spectrum to find a good compromise between the number of "walkers", the number of iterations per walker, and the total computational time. We found that convergence was reached after $540 \pm 100$ iterations (also called the burn-in size), such that we ensured convergence in an acceptable computational time in each case by setting a maximum of 2200 iterations for each of the 32 walkers. The whole bandwidth was used in the fitting procedure. We selected the 30 best fits obtained from these iterations to demonstrate the fitting quality obtained with our parametrized line shape (see Fig. 1).

\section{Appendix D: Systematic and random error analysis}

There are several sources of possible systematic and random errors at the various wind speed retrieval stages. The first, obvious, cause of uncertainty is the spectral noise. The fact that we used spectral resolutions of $103 \mathrm{~m} \mathrm{~s}^{-1}$ for HCN may seem contradictory to our goal of detecting winds with expected velocities on the order of $100 \mathrm{~m} \mathrm{~s}^{-1}$ or less. However, the observation of the full line profile with high $\mathrm{S} / \mathrm{N}$ enables us to fit the whole line profile and derive the line center position with an accuracy that exceeds the spectral resolution by using tens to hundreds of spectral points in the fitting procedure. The uncertainty on the retrieved wind velocity, $v_{\text {wind }}$, can be estimated by the following empirical formula:

$\Delta v_{\text {wind }} \sim \frac{F W H M}{S / N \times \sqrt{F W H M / \Delta v}} \times \frac{c}{v_{0}}$,

where FWHM is the full width at half maximum of the line, $\mathrm{S} / \mathrm{N}$ is the signal-to-noise ratio per independent spectral channel, $\Delta v$ is the spectral channel width, $c$ is the speed of light, and $v_{0}$ is the line rest frequency. For HCN, the FWHMs of the lines lines differ significantly between the low-to-mid and high latitudes: $8 \mathrm{MHz}$ and $2 \mathrm{MHz}$, respectively. The resulting estimates of $\Delta v$ are $\sim 30 \mathrm{~m} \mathrm{~s}^{-1}$ and $\sim 15 \mathrm{~m} \mathrm{~s}^{-1}$, respectively, and the MCMC fitting procedure gives $\Delta v \sim 20 \mathrm{~m} \mathrm{~s}^{-1}$ and $\sim 10 \mathrm{~m} \mathrm{~s}^{-1}$, respectively.

Another source of random error is the continuum subtraction before the MCMC fitting procedure. Even though the HCN line is located in the far wing of the $\mathrm{NH}_{3}$ line at $572 \mathrm{GHz}$, the continuum should be flat within $\sim 0.1 \%$ over the observed bandwidth. However, the continuum subtraction applied in the uvplane within CASA before the imaging stage sometimes resulted in a non-flat continuum on the resulting spectra. We proceeded with an additional subtraction of a first order polynomial from the spectra to flatten their continuum. This process implies a slight shift of the line center. This effect is relatively independent of the latitude, and we estimate from our simulations that the added uncertainty on the velocity is lower than $10 \mathrm{~m} \mathrm{~s}^{-1}$ on the $\mathrm{HCN}$ wind speeds. We quadratically added this uncertainty to the MCMC uncertainty.

At a given latitude, when the wind speed is lower than the noise level, one would expect the eastern and western limb wind speeds to be centered on the zero-velocity line. On the contrary, when a zonal wind is present, the east and west wind curves should in principle mirror each other. However, we initially found that the curves suffered a negative offset of $\sim 30 \mathrm{~m} \mathrm{~s}^{-1}$ in the mid-latitudes (i.e., where there is no clear detection). In what follows, we discuss the two sources of systematic errors that could be the cause of this offset and detail how we treated them.

\section{D.1. Jupiter radial velocity correction}

The first obvious systematic error that could cause an overall shift of the wind speeds concerns the accuracy of the Jovian radial velocity correction. It is made within CASA by using a JPL/Horizons ephemeris. The correction is applied with the accuracy of the ephemeris table at the level of individual integrations (i.e., every few seconds) by interpolating linearly between table entries. The geocentric radial velocity is tabulated with an accuracy of $10^{-8} \mathrm{UA} \mathrm{day}^{-1}$, with one entry per day. By interpolating to a time within the range of our observations, we find that the systematic error on the radial velocity correction is $<1 \mathrm{~m} \mathrm{~s}^{-1}$.

\section{D.2. Offset of the planet center position on the spectral maps}

The wind-induced Doppler shifts from each limb measurement are retrieved by subtracting the planetary rotation velocity projected along the LOS to the fitted central frequency of the lines. It is important to account for the elliptical shape and orientation of the synthetic beam to compute the line shift induced by the 


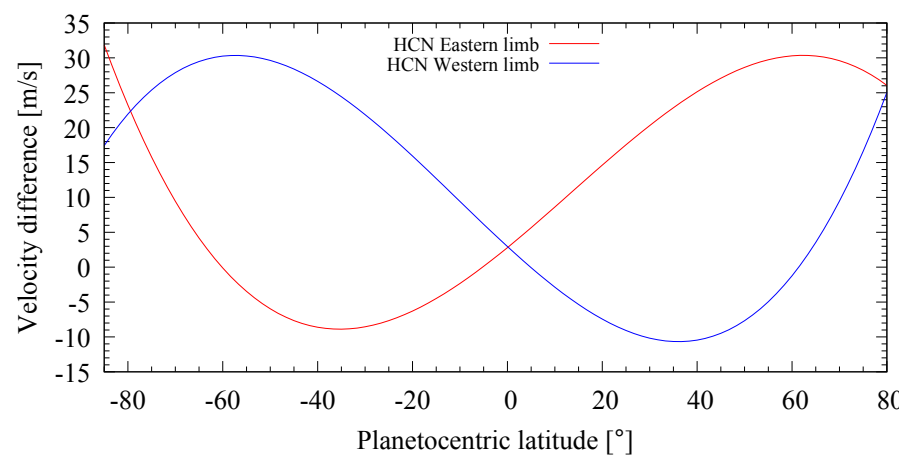

Fig. D.1. Effect of pointing uncertainty on wind speed retrieval. The velocity difference as a function of latitude between two sets of LOS velocity retrievals is shown. The set in which the planet is assumed to be centered in the field-of-view is subtracted from the other, in which we applied the position offset as determined from the analysis of the continuum. Results are shown for both limbs.

planetary rotation. We took the System III rotation period of Jupiter. To compute the spectral shift caused by the planet's solid rotation for each extracted spectrum, we accounted for the projection of the rotation velocity vector on the LOS and the convolution by the synthetic beam. After self-calibrating the data to best center Jupiter on the image, there can still be a small center offset of up to one-tenth of a synthetic beam due to interferometric seeing. This affects our planet rotation speed subtraction process. To correct for this, we used the continuum images at $354 \mathrm{GHz}$ to constrain the location of the center of the planet. We found that the planet center was shifted by $53 \pm 5$ mas in right ascension and $-32 \pm 6$ mas in declination. The curves presented in Fig. 2 take these offsets into account. The remaining uncertainty, equivalent to $5 \mathrm{~m} \mathrm{~s}^{-1}$, is caused by continuum variability in the millimeter wavelength range (de Pater et al. 2019). This random error was added quadratically to the previous ones. When compared to the ideal case of a wind speed retrieval with a perfectly centered planet, this position shift results in a velocity difference as a function of latitude that is not uniform because of the position angle of the planet. While an east-west equatoraligned offset shifts the two wind curves in the same direction, a north-south offset distorts the overall shifts. The difference is obtained by subtracting the two retrieved wind curves from one another, which is shown in Fig. D.1.

\section{Appendix E: Modeling the spectral effect of a constant counterrotation wind inside the auroral ovals}

To fit the northern and southern auroral wind speeds of Fig. 2 (top), we simulated the Doppler shifts induced by constant winds within the auroral ovals on the spectral lines. We took the radiative transfer model from Cavalié et al. (2019) and the HCN vertical profiles derived from the line shape analysis: from $60^{\circ} \mathrm{S}$ to $50^{\circ}, \mathrm{HCN}$ was set constant to $1 \mathrm{ppb}$ for $p_{0}<5 \mathrm{mbar}$, and to zero at higher pressures. For latitudes lower than $60^{\circ} \mathrm{S}$ and higher than $50^{\circ} \mathrm{N}$, HCN was set constant to $1 \mathrm{ppb}$ for $p_{0}<0.1 \mathrm{mbar}$, and to zero at higher pressures. For the auroral ovals, we took the oval's inner and outer edges as defined by Bonfond et al. (2012) as a starting point and set constant wind speeds within these edges. We then proceeded as follows. First, we conducted a simulation of spectral lines at infinite spectral and spatial resolutions on a highly sampled planetary grid. Second, we applied a spectral shift induced by the planet rotation for all LOS. Third, we applied a spectral shift induced by the LOS component of the constant wind when the LOS intercepted the auroral oval. Fourth, we convoluted the spectra spatially by the ALMA beam and spectrally for each studied pointing. Finally, we measured the line center frequency for each studied pointing and subtracted the contribution of the planet rotation, which was derived from a similar simulation in which there was no auroral wind.

On the southeastern limb, the oval is at $-72^{\circ}$ and is $\sim 3^{\circ}$ wide in the model from Bonfond et al. (2012). Using this definition of the oval does not provide a good fit to the data. The wind peak is too narrow, as shown in Fig. E.1. The northern oval is also too narrow to provide the large wind peak seen at $57^{\circ} \mathrm{N}$. We had to extend the southern oval by $1.5^{\circ}$ poleward and $2^{\circ}$ equator-ward to improve the fit to the data. On the northern oval, and for similar reasons, we had to shift the southern inner edge northward by $\sim 5^{\circ}$. These adjustments to the size of the northern and southern ovals may translate from a more extended oval episode of the aurora. We found that wind speeds of $300 \mathrm{~m} \mathrm{~s}^{-1}$ in the northern oval and $370 \mathrm{~m} \mathrm{~s}^{-1}$ in the southern oval, both in counterrotation, provide us with a relatively good fit to the data given the simplicity of the auroral oval wind model. The LOS projected components of the auroral oval wind at infinite spatial resolution are presented in Fig. E.2, and the comparison with the wind measurements is shown in Fig. E.1. 


\section{Northern polar region}
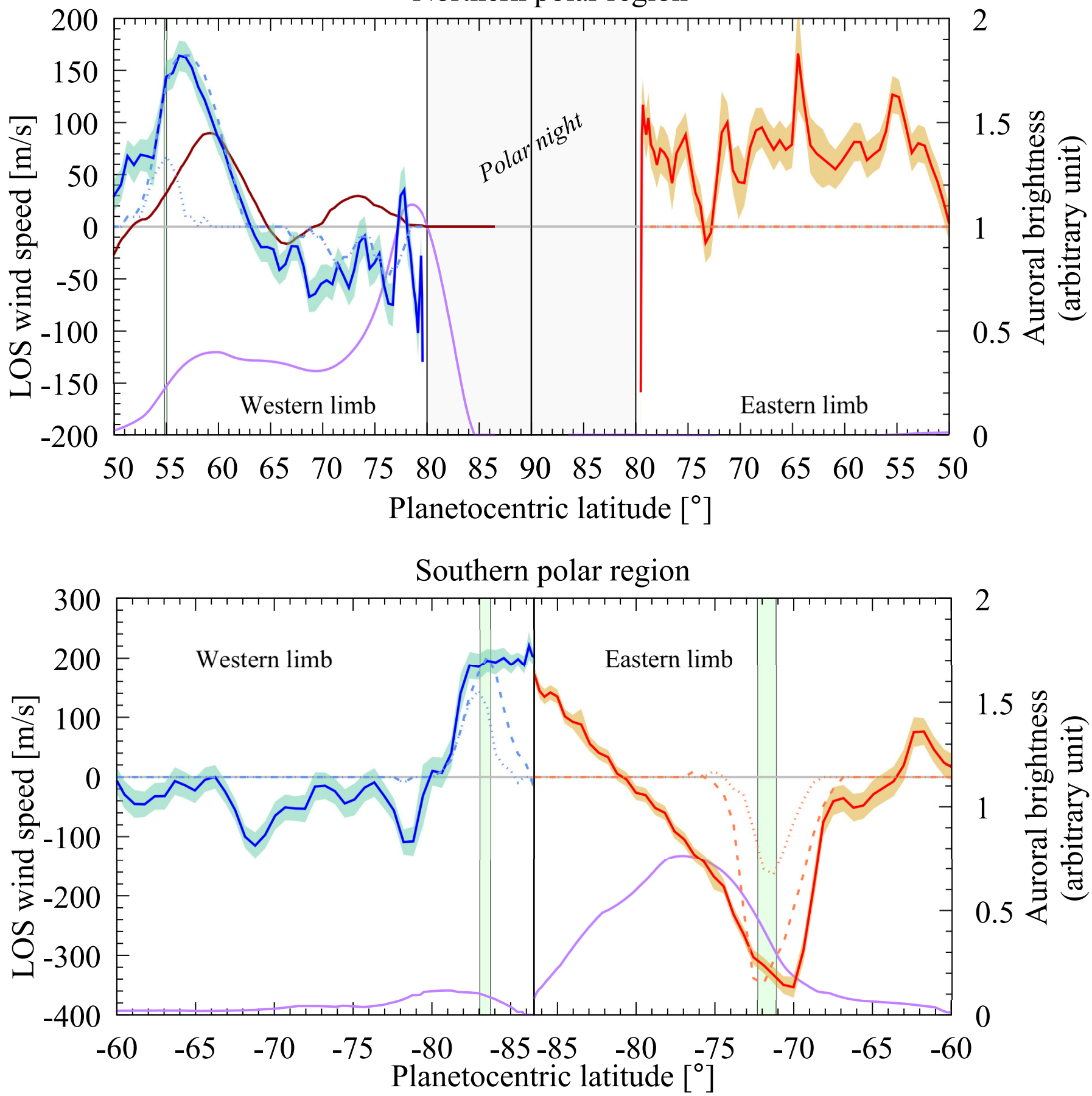

Fig. E.1. Comparison of models with observations: comparison between LOS velocities measured at 0.1 mbar with ALMA (same color code as in Fig. 2, top) and simulation results. A model with constant counterrotation winds within the ovals as defined by Bonfond et al. (2012) results in too narrow wind peaks, as shown with the dotted light blue and orange lines, regardless of the wind speed. The dashed light blue and orange lines are produced with constant counterrotation winds within the auroral ovals of $300 \mathrm{~m} \mathrm{~s}^{-1}$ (north) and $370 \mathrm{~m} \mathrm{~s}^{-1}$ (south) in the case of extended ovals, as described in the main text. The latitudes swept by the $M=30$ footprints of the Connerney et al. (2018) magnetic field model are plotted with green stripes. The wind peaks are found within $1-2^{\circ}$ of these footprints. The statistical UV brightness model (purple line) derived from the observations of Clarke et al. (2009) and the ionospheric winds (brown line; speeds divided by five on the plot for an easier comparison) derived from the $\mathrm{H}_{3}^{+}$infrared observations of Johnson et al. (2017) are also included for a qualitative comparison with our measured wind speeds. Both UV brightness and $\mathrm{H}_{3}^{+}$wind curves have been degraded to the ALMA spatial resolution. The data from Johnson et al. (2017) are taken from their Fig. 8f. The northern auroral region is in the top panel, and the southern auroral region is in the bottom panel. 

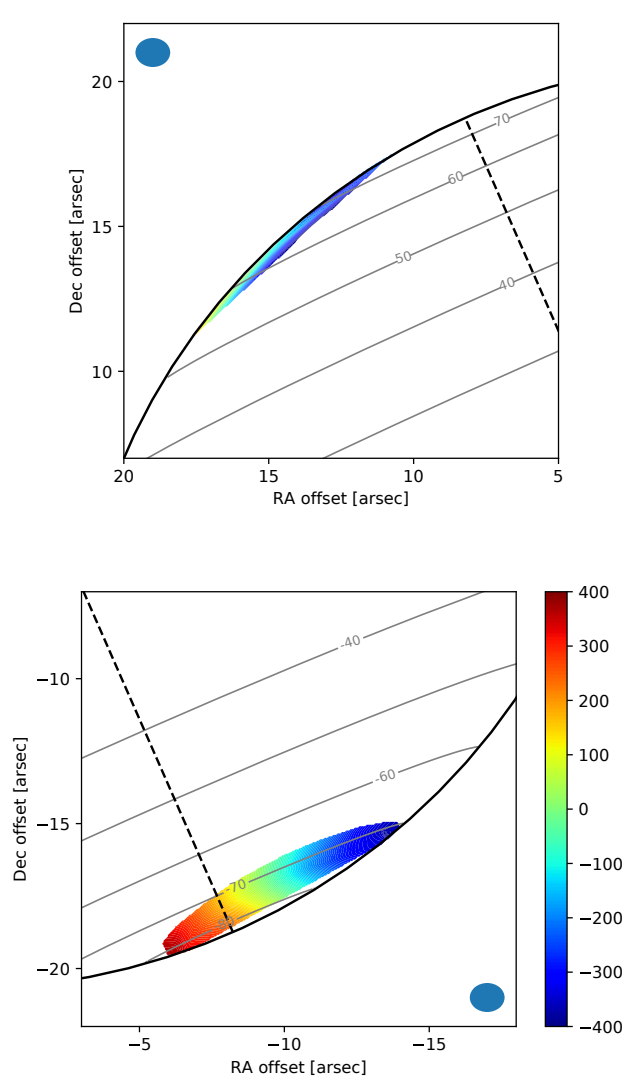

Fig. E.2. Auroral oval wind model: LOS component of the winds in the auroral ovals at infinite spatial resolution, assuming a counterrotation wind speed of $300 \mathrm{~m} \mathrm{~s}^{-1}$ in the northern aurora (top) and $370 \mathrm{~m} \mathrm{~s}^{-1}$ in the southern aurora (bottom). The ALMA synthetic beam size is displayed with a filled blue ellipse, and the planetocentric latitudes are indicated.

\section{Appendix F: Comparison with UV brightness distribution and IR ionospheric wind data at auroral latitudes}

Figure E.1 also presents a comparison of the wind speeds measured at $0.1 \mathrm{mbar}$ in the polar regions with the brightness of the aurora seen in the UV. There were no UV observations performed simultaneously to our ALMA observations. We thus chose to use the statistical UV aurora brightness model derived from the observations of Clarke et al. (2009) and set it to the geometrical configuration of our ALMA observations, as presented in Fig. F.1. On the northwestern limb, the HCN wind peak at $57^{\circ} \mathrm{N}$ is relatively well collocated with the first UV peak $\left(59^{\circ} \mathrm{N}\right)$, which corresponds to the southernmost part of the oval in our field-of-view. We see no obvious wind peak coinciding with the northernmost part of the oval in the field-of-view, which also corresponds to the most intense UV peak (at $88^{\circ} \mathrm{N}$ ), because this latitude is at the limit of the polar night region and wind measurements are therefore much noisier. At the southeastern limb, there is a larger offset of $7^{\circ}$ between the position of the oval, as determined from the UV brightness peak $\left(77^{\circ} \mathrm{S}\right)$, and the peak seen in the wind speeds $\left(70^{\circ} \mathrm{S}\right)$. Finally, we also find an offset of $2-3^{\circ}$ between the oval and the wind peak on the southwestern limb. The collocation of the wind speed peaks and the UV auroral brightness peaks of this statistical model is thus quite limited. Furthermore, there seems to be no correlation between wind speed and UV brightness within the oval. However, the Jovian UV aurorae have shown a significant degree of variabil-

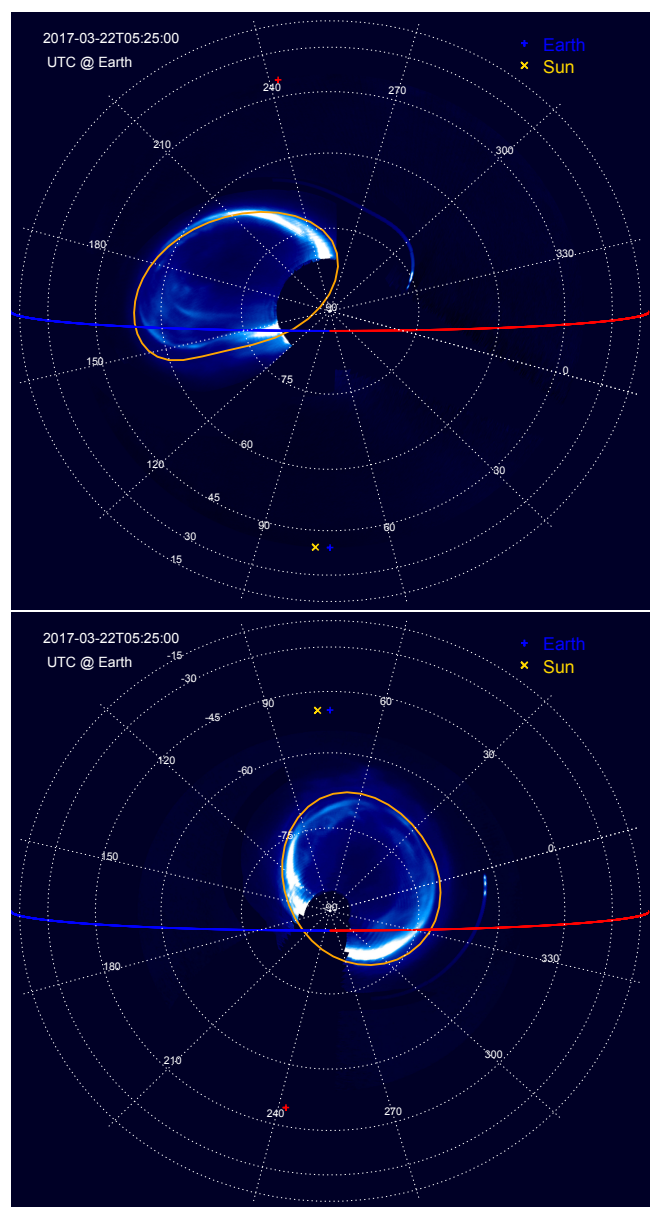

Fig. F.1. Ultraviolet aurora models at the time of the ALMA observations. Top: northern polar projection of the brightness of the statistical UV aurora in the configuration of the ALMA observations. The red and blue lines represent the east and west terminators. The orange lines show the $M=30$ footprints of the magnetic field model from Connerney et al. (2018). The subsolar and sub-Earth points are indicated with yellow and blue crosses, respectively. The red cross indicates the CML of the Johnson et al. (2017) measurements of ionospheric winds. Bottom: southern polar projection of the brightness of the statistical UV aurora in the configuration of the ALMA observations.

ity over all kinds of timescales (e.g., Grodent et al. 2003). The fact that we had to increase the width of the southern oval to improve the fit to our data (see Fig. E.1) seems indicative of an episode of extended ovals.

Juno-UVS observations (Gladstone et al. 2017) have shown that the position of the main ovals is well marked by the $M=$ 30 footprints of the magnetic field model from Connerney et al. (2018). Figure E.1 shows that we observe the HCN polar wind peaks within $1-2^{\circ}$ of these footprints.

In Fig. E.1, we also compare our data with the ionospheric winds derived from $\mathrm{H}_{3}^{+}$observations by Johnson et al. (2017). For this comparison to be as accurate as possible, we only took the data from their Fig. 8f; the CML was $\sim 240^{\circ}$, which places the position of the terminator within $10^{\circ}$ of the ALMA observation geometry. Here, we found a small offset of $3^{\circ}$ between the counterrotation $\mathrm{HCN}$ and $\mathrm{H}_{3}^{+}$wind speed peaks, as shown in Fig. E.1.

Because of the aurora variability, we need simultaneous observations to fully assess how well the stratospheric auroral oval winds detected in this work are collocated with the auroral UV brightness peaks associated with the ovals, as well as with the higher altitude auroral winds seen with $\mathrm{H}_{3}^{+}$. 